\title{
EDITORIAL \\ Obesity stigma: a persistent problem, a possible solution
}

International Journal of Obesity (2013) 37, 1413-1414; doi:10.1038/ ijo.2013.34

In this issue of the journal, Puhl et al. ${ }^{1}$ have published a study about an important topic - the stigma about obesity and weight bias. This and other research groups have already uncovered the deep rooted negative bias in society against obese individuals. Lacking any legitimate basis, and despite contrary evidence, ${ }^{2}$ obese people are widely perceived to be lazy, lacking will power, undisciplined, or unintelligent. Sadly, even health care providers, the very people expected to counsel and treat obesity, are prejudiced against obese individuals. Now, Puhl et al. ${ }^{1}$ report that physicians themselves could be the target of weight stigma from their patients. Puhl et al. ${ }^{1}$ conducted an online survey of 358 participants who were randomly assigned to questionnaires about normal weight, overweight or obese physicians. As a result, it was found that patients were less likely to trust overweight and obese doctors or follow their advice. They were also less likely to select overweight or obese physicians and more likely to switch doctors who were obese. Those participants with greater fat-phobia were more likely to trust normal weight physicians or switch obese physicians.

It is unfortunate that a physician's own weight could impede the reception of medical advice. However, considering the high prevalence of weight bias in society, it would be surprising if physicians were immune from it. About $48 \%$ of the survey participants were themselves overweight or obese and $48 \%$ were normal weight. Although normal-weight individuals were somewhat over-represented in the sample compared with the national prevalence, weight bias against physicians was displayed regardless of the body weight of the participants. This is another indication that even those suffering from weight issues themselves do not view obesity differently.

Substantial evidence has accumulated about obesity stigma. However, simply documenting stigma is not likely to reduce it. It is inadequate to state that weight bias is bad, if not explained why and how it is ill founded. Obesity stigma seems to stem from a misconception that obesity is a choice, and its successful treatment is under complete volitional control. This misconception has possibly lead to, and will continue to propagate outrageous consequences, such as refusing to serve obese individuals in restaurants, financial penalties on the obese individuals proposed by lawmakers and even a reduction in obesity research funding by federal agencies (http://www.medscape.com/viewarticle/743363_5).

Although many obesity researchers are aware of the multiple contributors of obesity that are beyond personal control, the concept has not yet adequately permeated the general public's consciousness. Moreover, the promotion of naive and simplisticsounding strategies touted for obesity prevention and treatment must add to this prejudice. Many messages about weight management single out a specific food or drink to eat or avoid, or simple physical activity tasks to undertake. Many such simplistic measures are in fact myths, ${ }^{3}$ and more importantly distort and downplay the tremendous amount of effort and commitment that a successful weight management requires. Therefore, it is little surprise that prejudices exist against a condition perceived to be a simple matter of choice. In fact, in a study, participants increased anti-fat prejudice when informed that obesity is due to controllable reasons. Whereas, those who were informed about the 'uncontrollable' reasons for obesity, reduced their prejudice. ${ }^{4}$ Thus, to reduce the anti-fat bias in general population, it may be necessary to modify those ineffective public health guidelines or policies and messages in popular media, which tend to downplay the efforts required to control obesity.

Although weight bias is undesirable in any interaction, it carries additional implications in a doctor-patient relationship. Physicians and patients both harbor a stigma against each other's body weights, which must add complexity particularly to the already challenging treatment of obesity. Patients, including those who are obese, trust normal-weight doctors more than obese doctors. However, those normal weight doctors are more likely to be prejudiced against obese patients. It is unknown whether these perceptions actually influence outcomes of obesity treatment. Also, as pointed out by Puhl et al., ${ }^{1}$ it remains to be determined whether the suboptimal reception of medical advice by patients is limited to only matters of weight management or extends to other health conditions.

Physicians have an additional responsibility in this issue. Patients who receive weight management advice from their health care professional, are more likely to attempt weight loss. ${ }^{5}$ Yet, barely $40 \%$ of obese patients are advised by their physicians to lose weight. ${ }^{5}$ Therefore, physicians should discuss weight-loss strategies with patients, as needed. Many surveys show that nearly half of the physicians feel underprepared and ill equipped to treat obesity. This needs to change. Physicians with normal weight seem more confident to counsel about weight. ${ }^{6}$ However, the study by Puhl et al. ${ }^{1}$ should not be wrongly interpreted that overweight or obese physicians should 'shape up or ship out'. Far from it, this study underscores the need for a candid and evidence-based conversation with patients about their body weight and health risks associated with excess body weight, regardless of a physician's own body weight.

The stigma against physician's body weight may decrease, if the true complexity of obesity is revealed to patients. It is imperative that physicians candidly explain to their patients the limitations of current weight-loss strategies and the efforts involved, and present a realistic probability of weight-loss maintenance. Such a dialog may be the key to leading patients and the general public to a better and factual understanding of this complex medical condition, and may alleviate the anti-fat prejudice of physicians and their patients.

In summary, Puhl et al. ${ }^{1}$ once again remind us of the pervasive stigma against obesity. Messages that simplistically and selectively emphasize volitional control of body weight and ignore or drown out information about the non-volitional contributors of obesity, probably foster this stigma. Weight bias not only hurts individual victims socially, but also hurts society, by influencing policy decisions about health, research and patient care, and in turn distracts from finding effective solutions. To reduce the stigma of obesity, concerted efforts are required to more effectively convey the non-volitional aspects of weight gain and weight loss to general population. Informed physicians and other health care professionals, media and researchers, all could have a role in such a campaign to reduce weight bias in society.

\section{CONFLICT OF INTEREST}

The author declares no conflict of interest. 
NV Dhurandhar

Pennington Biomedical Research Center, Baton Rouge, LA, USA E-mail: Nikhil.Dhurandhar@PBRC.EDU

\section{REFERENCES}

1 Puhl RM, Gold JA, Luedicke JA, DePierre JA. The effect of physicians' body weight on patient attitudes: implications for physician selection, trust and adherence to medical advice 2013; 37: 1415-1421.

2 Roehling MV, Roehling PV, Odland LM. Investigating the validity of stereotypes about overweight employees. Group Organ Manage 2008; 33: 392-424.
3 Casazza K, Fontaine KR, Astrup A, Birch LL, Brown AW, Bohan Brown MM et al. Myths, presumptions, and facts about obesity. N Engl J Med (Research Support, NIH, Extramural) 2013; 368: 446-454.

4 O'Brien KS, Puhl RM, Latner JD, Mir AS, Hunter JA. Reducing anti-fat prejudice in preservice health students: a randomized trial. Obesity 2010; 18: 2138-2144.

5 Galuska DA, Will JC, Serdula MK, Ford ES. Are health care professionals advising obese patients to lose weight. JAMA 1999; 282: 1576-1578.

6 Zhu D, Norman IJ, While AE. The relationship between health professionals' weight status and attitudes towards weight management: a systematic review. Obes Rev 2011; 12: e324-e337. 